\title{
Health technology assessment in low- and middle- income countries: a case study of trastuzumab for early and locally advanced HER-2 positive breast cancer in Tunisia
}

Pascale Dequen-0'Byrne ( $\sim$ pascale.dequen@visibleanalytics.co.uk)

Visible Analytics Ltd https://orcid.org/0000-0002-5120-8675

Mark Sculpher

University of York Centre for Health Economics

Neil Hawkins

Visible Analytics Ltd.

Juliette C Thompson

Visible Analytics Ltd.

Keith R Abrams

Visible Analytics Ltd.

\section{Research}

Keywords:

Posted Date: March 3rd, 2021

DOI: https://doi.org/10.21203/rs.3.rs-264006/v1

License: (c) (1) This work is licensed under a Creative Commons Attribution 4.0 International License.

Read Full License 


\section{Health technology assessment in low- and middle-income countries: a case study of trastuzumab}

for early and locally advanced HER-2 positive breast cancer in Tunisia

Pascale Dequen-O'Byrne MSc, PhD¹, Mark Sculpher MSc, PhD², Neil Hawkins MSc PhD'1,3, Juliette C. Thompson $\mathrm{BSc}^{1}$, Keith R. Abrams MSc PhD ${ }^{1,4}$

1 Visible Analytics Ltd., Oxford, UK.

2 Centre for Health Economics, University of York, York, United Kingdom.

3 Health Economics and Health Technology Assessment, University of Glasgow, Glasgow, UK

4 Department of Health Sciences, University of Leicester, Leicester, UK

\section{Corresponding author:}

Pascale Dequen-O’Byrne

Visible Analytics Ltd.

3 Kings Meadow

Oxford, Oxfordshire

OX2 0DP

UK

pascale.dequen@visibleanalytics.co.uk

\section{Sponsors:}

This work was commissioned by Roche Tunisia SA, but the funder had no role in reviewing or commenting on the content of the manuscript. Professor Abrams is partially supported as a UK National Institute for Health Research (NIHR) Senior Investigator Emeritus (NF-SI-0512-10159). The views expressed in this publication are those of the author(s) and not necessarily those of the NHS, the National Institute for Health Research, or the Department of Health. 


\section{Background}

In 2014, the World Health Organization (WHO) reiterated the critical role of health intervention and technology assessment to inform priority setting and resource allocation, reduce inefficiencies, and ultimately achieve universal health coverage [1,2]. In the past decade, global stakeholders from all sectors of healthcare have embraced evidence-based health policy development and decision-making, resulting in a growing number of initiatives to promote health technology assessment (HTA) particularly in low- and middle-income countries (LMICs). Initiatives such as the Guide to Health Economic Analysis and Research (GEAR), Decide-Health Decision Hub, the International Decision Support Initiative (iDSI), Thailand's Health Intervention and Technology Assessment Program (HITAP) international unit or the National Institute for Health and Care Excellence (NICE) international services provide a collection of online resources and tools to share, establish and strengthen HTA capacity worldwide $\left.{ }^{2-5}\right]$.

A key principle of HTA, widely acknowledged by international agencies—both established and emerging, is the use of scientifically rigorous and evidence driven methodology to ensure quality and consistency of assessments, over time and across health interventions, and to present these in a transparent manner [6]. In May 2020, the International Network of Agencies for Health Technology Assessment (INAHTA) released a new definition of HTA which notes the process of HTA should be: "formal, systematic and transparent, and uses state-ofthe-art methods to consider the best available evidence" [7]. However, to date no universally comprehensive framework or 'how-to' methods guide for HTA currently exist, or indeed is ever likely to exist, and the practice of HTA remains intrinsically shaped by a range of country-specific factors, policy agendas and institutional contexts, as well as, social and cultural values [ $\left.{ }^{8}\right]$. In addition, inherent challenges of implementing HTA methods in LMICs, namely limited resources, capacity and capabilities in the key disciplines of HTA, and potential lack of local data to inform decision-making, can curtail efforts by new agencies to adhere to HTA best practice $\left[{ }^{9,10}\right]$.

\section{Case study: trastuzumab in Tunisia}

In Tunisia, breast cancer is the primary cancer in women and represents the most common cause of cancer mortality [11,12]. The standardised incidence of breast cancer in Tunisian woman has nearly tripled since 1994, reaching $50.17 / 100,000$ persons years in 2017 [ ${ }^{13,14}$ ]; it is projected to grow even further in coming years to eventually match the incidence of HICs. HER-2 positive breast cancer patients represent between 15 to $25 \%$ of all cases [15]. Trastuzumab—a targeted cancer therapy—was granted market authorisation by Tunisian regulatory authorities for early or locally advanced breast cancer in 2008. Although it is considered the 'gold' standard in the adjuvant setting and already used in clinical practice in Tunisia, its funding in its licensed indication represents the 
largest drug cost burden for the national health service, raising concerns regarding its cost-effectiveness and 'economic acceptability' [13,15].

The health care system in Tunisia includes both public and private sectors; however, the publicly run Caisse Nationale d'Assurance Maladie (CNAM) —-the compulsory national social health insurance schemeprovides care for the majority of the population $\left[{ }^{16}\right]$. In 2013 , the WHO reported more than $90 \%$ of the Tunisian population were covered through the CNAM or the free medical assistance programme for the most vulnerable $\left[{ }^{16,17}\right]$. Public sector coverage under the CNAM includes health centres providing primary care, district and regional hospitals, and university hospitals.

In May 2017, INEAS was commissioned to conduct an HTA of trastuzumab for early and locally advanced HER2 positive breast cancer; in July 2018, it published its first guidance online [ $\left.{ }^{15}\right]$. The scope of the INEAS assessment was to conduct a pharmacoeconomic analysis and evaluate the net clinical benefit of trastuzumab for early and locally advanced HER2 positive breast cancer. Their evaluation included: (1) a review of the literature to summarise the net clinical benefit of trastuzumab compared with standard chemotherapy; (2) a comparison of international clinical practice guidelines recommendations; (3) an international drug price comparison; (4) a survey of international HTA agencies on the assessment of trastuzumab; and (5) a CEA.

\section{Objectives and methods}

We use the case study of the first published HTA by INEAS—-trastuzumab (Herceptin®) in early and locally advanced HER2 positive breast cancer ${ }^{15}{ }^{15}$ — to critically compare the approach and methods used by an emerging HTA body to widely cited good practice guidelines in HTA [18-22], given likely resource and capacity constraints. The degree to which this is possible is limited by a country's willingness to share detailed descriptions of the methods used and results of their technology assessments. The Tunisian National Authority for Assessment and Accreditation in Healthcare (INEAS) was created in 2012 with an overarching goal to regulate the national health system by promoting quality and efficiency [www.ineas.tn/fr]. Within its newly acquired mandate, HTA is at the core of its mission. In this case, we refer to the WHO's definition of HTA as: "the systematic evaluation of properties, effects and/or impacts of health technologies and interventions" [ $\left.{ }^{1}\right]$. This multidisciplinary process of clinical and economic assessment is taken as distinct from the appraisal or decision-making, informed by this assessment, but which inevitably involves a range of other considerations and which is typically less transparent.

The HTA guidelines used were identified via the INAHTA website, the GEAR health economic evaluation guidelines database, and the ISPOR Outcomes Research Guidelines Index. These include the landmark report from the European Collaboration for HTA/Assessment of Health Interventions (ECHTA/ECAHI) project promoting 
best practice in undertaking and reporting HTA $\left[{ }^{18}\right]$, the Centre for Review and Dissemination (CRD)'s guidance for undertaking systematic reviews in health care $\left[{ }^{22}\right]$, the WHO guide to cost-effectiveness analysis $(\mathrm{CEA})\left[{ }^{23}\right]$ and the Gates Reference Case for Economic Evaluation [24]. Published methods guidelines from HTA agencies in low-, middle- and high-income countries (HICs) were also considered, such the Indonesian Health Technology Assessment Committee HTA guidelines [ $\left.{ }^{25}\right]$, the guidelines for HTA in Thailand $\left[{ }^{26}\right]$, and the NICE methods guide in England and Wales [20]. To date, INEAS has not published a methods or process guide for their assessments, and we did not identify any peer-reviewed guidelines tailored to the conduct of HTA within the region or specific to LMICs.

First, we summarise the clinical and economic evidence assessed by INEAS ${ }^{1}$. The critical review of the INEAS report is structured according to Busse et al. framework for HTA content and the key elements of HTA reporting, as presented in Table $\left.1{ }^{18}\right]$. Lastly, we provide recommendations for future practice drawing on lessons learnt from other HTA agencies in LMICs, and ongoing international research initiatives and collaborations to promote HTA methods worldwide.

\section{Table 1. Content of a Health Technology Assessment}

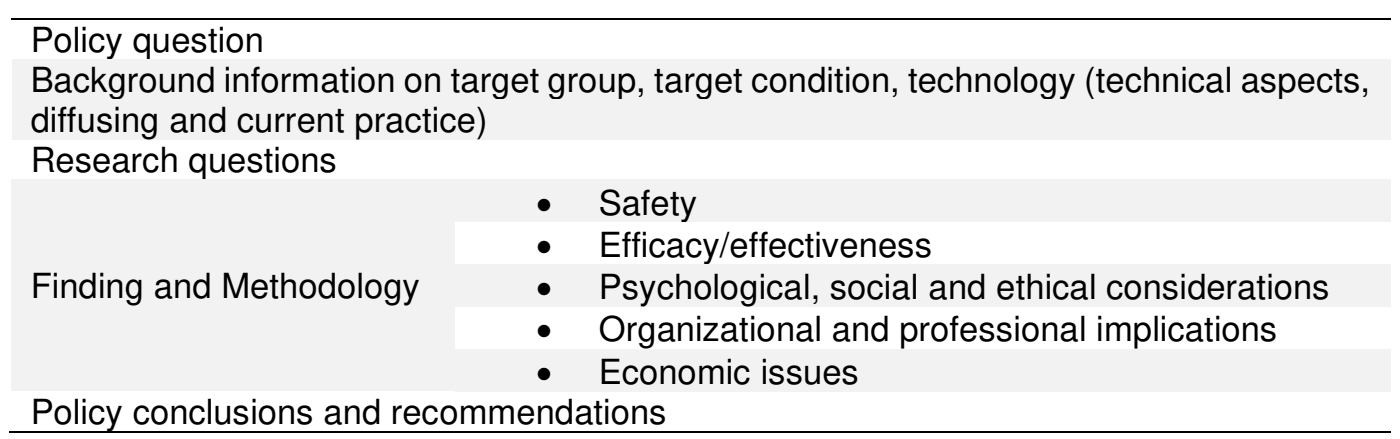

\section{Summary of trastuzumab clinical evidence}

The review of the clinical literature performed by INEAS was two-fold. In a first instance, assessors identified all relevant clinical practice guidelines for the treatment of HER2 positive breast cancer to compare international recommendations on the use of trastuzumab; second, they summarised the risk-benefit profile of trastuzumab in the indicated population. Two clinical guidelines were included for comparison with the STOMSociété Tunisienne d'Oncologie Médicale—protocol for trastuzumab, the recently updated NICE guidance for early and locally advanced breast cancer and the Scottish Intercollegiate Guidelines Network (SIGN) guidelines for the treatment of primary breast cancer [27-29]. The target population and treatment administration of trastuzumab across

\footnotetext{
${ }^{1}$ It should be noted that the full-text trastuzumab INEAS report is only available in French ${ }^{15}$, the original report was used in the critical review as one of the authors is French native and could translate, as necessary.
} 
the three guidelines aligned with the licensed indication, similar risks and contraindications were noted, and regular monitoring of cardiac function was recommended. Assessors presented the pooled results published in the Cochrane Systematic Review on trastuzumab containing regimens for early breast cancer, showing significant improvements in disease-free and overall survival for patients treated with trastuzumab as adjuvant therapy [30].

\section{Summary of trastuzumab economic evidence}

A systematic search of economic evaluations for trastuzumab in combination with standard chemotherapy compared with standard chemotherapy in early or locally advanced HER2 positive breast cancer was undertaken. The breadth of the search extended to six preferred online databases and the methodological quality of the included CEAs was assessed using two standard checklists: FLC 2.0 [lecturacritica.com] and Drummond's checklist for assessing economic evaluation [19].

No economic model, specific to the Tunisian health system perspective, was identified in the systematic review or developed de novo by INEAS or provided by the manufacturer. Instead, INEAS adapted a published Markov model by Pichon-Riviere et al. evaluating the cost-effectiveness, coverage, and accessibility of trastuzumab for patients with early HER2-positive breast cancer in seven Latin American countries $\left.{ }^{31(\mathrm{p})}\right]$. The modelling approach and input data used in the published CEA were summarised in the INEAS report. The safety and efficacy data, as well as the transition probabilities, were taken from the pivotal trastuzumab trial $\left[{ }^{32}\right]$; resource use and medical costs were informed by Latin American registries and hospital charges; and the quality of life valuation from two published utility studies [ ${ }^{33,34}$ ]. The drug costs for both trastuzumab and standard chemotherapy were estimated by INEAS, based on the list prices of trastuzumab in Tunisian dinar before 2016 and following a price reduction per vial in 2018. The incremental cost-effectiveness ratio (ICER) for Tunisia was then calculated (using the Equation 1, translated from the INEAS HTA report $\left.\left[{ }^{15}\right]\right)$.

\section{Equation 1. ICER calculation}

$$
I C E R_{\text {Tunisia }}=\frac{\left(\text { Total costs } S C T Z-\operatorname{Cost} T Z_{\text {Reference country }}+\text { Cost } T Z_{\text {Tunisia }}\right)-\text { Total costs } S C}{Q A L Y_{\text {Reference country }}}
$$

SC: standard chemotherapy; TZ: trastuzumab; SC TZ: standard chemotherapy + trastuzumab

INEAS found trastuzumab not cost-effective under the different pricing assumptions tested and based on their cost-effectiveness threshold calculations. Despite acknowledged caveats, INEAS described its conclusions as 
robust in the scenarios tested and consistent with results from other published CEAs. Moreover, they estimated that in order to be cost-effective at an upper threshold of up to three times Tunisia's gross domestic product (GDP) per capita, a discount of $78 \%$ on the current list price of trastuzumab would be required $\left[{ }^{15}\right]$.

\section{Critical assessment}

\section{HTA protocol and research questions}

The decision problem put forward by INEAS focused on a therapy already in common use in Tunisia, as opposed to a new health intervention. The stated rationale for this first assessment was the growing financial strain trastuzumab presented on the national health system and the need to explore the 'efficiency' of the drug in the given indication $\left.{ }^{15}\right]$. The report by INEAS clearly stated the policy question motivating the assessment and provided succinct, yet sufficient, background information on the condition and target population, technology, and current practice to contextualise the research questions posited. However, the report could have benefited from an a priori defined HTA protocol, describing explicitly how each stated objective was to be addressed, how and by whom [18,35]. In particular, which methodology was to be followed, based on which data were to be available, and how the retrieved evidence was to be synthesised. It should also be noted that despite the relatively high burden of disease and considerable percentage of health expenditure devoted to breast cancer in Tunisia [14,15]; at the time the assessment was published, the WHO had launched a pilot procedure for prequalification of biotherapeutic products including trastuzumab [36] and the market entry of biosimilars was likely to change the distribution and/or pricing of trastuzumab in the country.

\section{Findings and methodology: safety, efficacy/effectiveness}

In accordance with good practice guidelines, a systematic approach to identify relevant clinical evidence was presented by INEAS [18,20,22,37]. This included a pre-defined search strategy combining free-text and keywords, a stepwise study screening process by two independent reviewers, and a quality assessment of the included publications. Moreover, the reporting of the systematic search and review of clinical guidelines, demonstrated a willingness for transparency. The search strategies used by reviewers were included in an appendix and PRISMA diagrams were provided to illustrate the study selection process, as recommended by the CRD and NICE [20,22]. However, the inclusion and exclusion criteria used for study selection are not reported; a more detailed transcription of the search strategies annexed in the report-allowing for validation and/or replication by external reviewerwould have further improved transparency. 
Additional data sources cited by assessors, e.g. published systematic reviews and HTA/appraisals produced by other countries, to demonstrate the net clinical benefit of trastuzumab vs. standard chemotherapy were not identified systematically, and recent publications appeared to be omitted. For example, the STOM protocol compliance with international recommendations was only based on a comparison with two UK guidelines. International practice guidelines such as those published by the American Society of Clinical Oncology (ASCO) or European Society for Medical Oncology (ESMO) were not included in the review [ $\left.{ }^{38-41}\right]$.

The SIGN and NICE clinical guidelines formed the primary data source for the risk-benefit profile of trastuzumab and established its relative safety and efficacy compared with standard chemotherapy. However, it was unclear why the clinical literature review solely focused on identifying clinical practice guidelines. Drummond et al. suggest: "a comprehensive, well-conducted, [evidence-based medicine] review is a necessary first step in the HTA appraisal process" [35]; indeed, good practice principles favour systematic reviews and meta-analysis of randomised clinical trials (RCTs) often classified as the highest level of evidence to establish the relative safety, efficacy and effectiveness of technology compared to relevant comparators [18,20,22,37]. INEAS presented relative treatment effects for trastuzumab compared with standard chemotherapy in terms of disease free survival and overall survival; however, how the evidence transcribed from the trastuzumab Cochrane review and the patient populations in the included studies reflect that of the Tunisian context was not assessed [30]. The approach taken by INEAS is indeed more pragmatic, perhaps given time and/or resource constraints; however, this was not explicitly stated or justified by assessors and potential sources of bias in their approach were not reported.

\section{Findings and methodology: economic issues}

One of the key elements of the INEAS report was also acknowledged by assessors as its biggest limitation, that is the lack of a Tunisian-specific economic model and the absence of 'contextualised' data to inform a CEA. INEAS stated that despite an appeal to manufacturers to provide a CEA from the perspective of the Tunisian health system, an economic model was not submitted, and a de novo analysis could not be performed [15]. The conduct and reporting of the CEA search were more comprehensive than in the clinical section; PICO criteria were used to define the research question and inclusion/exclusion criteria were explicitly cited. The search methods used by INEAS were in line with CRD guidance on reviewing economic evaluations $\left[{ }^{22}\right]$. Some key search terms appeared to not have been used in the provided search strategy (e.g. economic evaluation or model, cost-benefit analysis), but this may have been a reporting issue with minimal impact on the search results. However, the review was restricted to CEAs performed in LMICs. This restriction was not substantively justified in the INEAS report and considerably 
reduced the number of included CEAs. Somewhat counterintuitively, the assessors later referenced CEAs and technology appraisals from HICs in their discussion of the CEA results.

The CEA methods and results reported by INEAS may not have adequately reflected the Tunisian health system perspective, nor accounted for local resource utilisation, costing, or quality of life measure [ ${ }^{23,35,42]}$. The comparability of direct medical costs between LMICs in Latin America and Tunisia was assumed by assessors, but no data were presented to validate this assumption $\left[{ }^{43}\right]$. Only the drug costs were adapted as per Equation 1. No sensitivity analysis or assessment of the uncertainty around the ICER could be performed beyond that published by Pichon-Riviere et al. $\left.{ }^{31}\right]$. The INEAS CEA raised the issue of the appropriateness of translating and transferring cost-effectiveness results across different jurisdictions. The Pichon-Riviere et al. model was selected by INEAS because it rated highly following the qualitative assessment of included CEAs; and assessors argued the direct medical costs included in the CEA for the five LMICs in Latin America were comparable to that of Tunisia [15,31]. The adaptation of economic models from other countries is relatively common in HTA practice; and can be much better use of limited resources and capacities compared to developing a local de novo model when one is not provided by the manufacturer or identified in the literature. However, some evidence can be considered more transferable across jurisdictions, such as treatment effects or utilities; a notable weakness here was the failure to adapt resource use and non-drug related costs to the Tunisian setting $\left.{ }^{43-45}\right]$. This probably would have required access to functional model. The economic evaluation of trastuzumab presented by INEAS would therefore be considered as weak evidence by most established HTA bodies, that is not to say non-informative for local healthdecision makers $[24,46]$.

\section{Policy conclusions and recommendations}

INEAS argued that drug costs were the key driver of trastuzumab's cost-effectiveness—or lack thereof—in Latin America and Tunisia. The price of trastuzumab in Tunisia was also tracked over time and internationally. Although the authors acknowledge the pricing of drugs in LMICs is important and timing, including the contentious price discrepancies between high- and LMICs and the healthcare funding dilemma often faced by the latter, a price comparison is not usually considered a key element of HTA. A budget impact model would have been more informative to address the research question of trastuzumab's 'efficiency' [ ${ }^{15}$; at least a presentation of the likely volume of patients who would be suitable for trastuzumab over time in Tunisia would have been useful.

Notwithstanding the methodological limitations and uncertainties surrounding the CEA, INEAS defended the relevance of their findings for Tunisia considering the magnitude of the 'inefficiency' of trastuzumab for the tested range of LMICs cost-effectiveness thresholds (CETs). The assessors did discuss the importance of how to 
appropriately judge the implications of the estimated ICERs for medicines in LMICs and the relevance of different CETs. Although there is still much debate and ongoing research on how to best define CETs in LMICs; recent work by Woods et al. and Ochalek et al. suggest that calculations of CET, such as the use of one to three times GDP/capita, might be too high as they do not adequately capture the opportunity cost in terms of the health benefits foregone because of interventions not provided [ $\left.{ }^{47-49}\right]$. This work would further support the conclusions from INEAS, that despite stated limitations of the economic evidence presented for trastuzumab in Tunisia, the magnitude of the negative ICERs outweighed the inherent uncertainty of the 'naïve' model adaptation.

Lastly, it should be noted that wider psychological, social, and ethical considerations, the patient perspective, as well as organisational and professional implications referenced in a number of good practice guidelines, were not explicitly reported in the INEAS assessment but may have been discussed by appraisers $\left[^{4,18}\right]$.

\section{Conclusions and recommendations}

We find the first HTA by INEAS to be an encouraging example of evidence-based practice to inform decision-making in an LMIC. In fact, we commend INEAS for making their assessment publicly available, allowing this critique, and providing a unique opportunity for shared learning. Overall, the conduct and reporting of the assessment of trastuzumab's net clinical benefit for early and locally advanced HER2 positive breast cancer in Tunisia was well-structured and transparent. However, our review also highlighted important limitations in the assessment of the CEA of trastuzumab compared with standard chemotherapy for the Tunisian health system. We acknowledge the learnings from this case study are limited as only reflect a single HTA report by one emerging HTA agency in a LMIC; however, our recommendations are more far-reaching and could be generalisable to other LMICs. Following their survey of researchers who have experience in conducting economic evaluations in LMICs, Luz et al. find similar technical issues were reported across a number of assessments: "the lack of quality local clinical data, poor reporting, insufficient data to conduct the analysis from the chosen perspective (i.e. the lack of cost data), lack of context-relevant standards for economic evaluation, and lack of local health-related preference data, respectively."10

Adapting or borrowing evidence across countries and HTA agencies can be a practical and efficient 'shortcut' to conducting HTA in LMICs, but it may not provide sufficient context to answer the policy and research questions set out by local agencies [50]. It may be misleading to assume that relative treatment effects are transferable, as the majority of randomised clinical trials for a particular condition may not be undertaken in LMICs and case-mix might differ between HICs and LMICs [ ${ }^{51}$. However, it may be challenging to develop or identify evidence regarding relative treatment effects in the local context. More important, resource utilisation, unit costs and 
quality of life data (and utilities using country-specific valuation sets) may differ substantially between LMICs for societal and cultural reasons; therefore care must be taken when comparing model inputs across jurisdictions, and ideally local data should be used [9,44,52].

The paucity of local data, or difficulties to obtain it, are well documented barriers to the implementation of HTA in LMICs [9,40]. Efforts to promote local data collection, especially through collaboration, should be prioritised. Considering the resource and time implications, obstacles to the gathering or sharing of data already being collected (e.g. cancer registries, medical cost data) could be removed; issues with missing or poor quality data should be addressed; or the use of technology (e.g. Electronic Health Records, especially in upper MICs) could generate local evidence both on clinical outcomes, resource use, etc. A number of LMICs are starting to develop frameworks for routine data collection and analysis of existing databases, such as medicines/healthcare utilisation and costs, following the example of countries such as Brazil or South Africa [ $\left.{ }^{53-57}\right]$. Gaining a better understanding of drug utilisation patterns and expenditures would also help prioritise key areas for HTA and resource allocation, which according to Jakupi et al. and Kivoto et al. is an essential first step before initiating HTAs [ $\left.{ }^{58,59}\right]$.

The challenges of local data collection also raise an important question regarding the 'burden of proof' and who bears the cost of making the case for the funding of a new technology, in the broader sense of, generating relevant and generalisable evidence, capturing contextualised data and modelling. In certain countries, the manufacturer is responsible for submitting this case for newly developed technologies. The degree to which manufacturers will be willing to submit assessments will depend on their own local resources and their perceived return on investment in such submissions. One consideration will be how the local agencies proceed if manufacturers decide not to make a submission. In this case study, INEAS decided to pursue the assessment using published evidence. It should also be borne in mind that for many technologies, such as those that no longer enjoy patent protection, there may not be a single identifiable manufacturer with a strong incentive to develop a submission.

The WHO and iDSI promote multiple stakeholder engagement as a tool to build capacity to support HTA $\left[{ }^{1,60}\right]$. The HTA process should be viewed by all stakeholders as a learning continuum allowing for capacity building, resource training, and re-assessments of health technologies over time. Emerging and newly established HTA agencies could consider formalising HTA protocols to encourage stakeholder engagement, assign tasks and impose timelines [ ${ }^{61,62}$. As exemplified by this review and others, such as the Ghanaian HTA study of the costeffectiveness of antihypertensive medicine; there are lessons to be learnt from the experiences and 'growing pains' 
of emerging HTA bodies when implementing HTA frameworks, capacity building, and engaging with multiple stakeholders [ $\left.{ }^{62}\right]$.

Improving communication and the sharing of information and resources could be a catalyst to the implementation of HTA in LMICs. Tantivess et al. reflect on the role NICE International and HITAP played in the HTA capacity development in Myanmar, finding: "[the] continuous support from international partners is indispensable for keeping the momentum of HTA introduction" [ $\left.{ }^{4}\right]$. Despite relying heavily on the Latin American experience and economic evaluation, INEAS disclosed a number of collaborations with other HTA agencies and a range of experts and stakeholders; in addition, to surveying international HTA bodies regarding previous assessments of trastuzumab in their respective jurisdictions [ $\left.{ }^{15}\right]$.

Other examples of initiatives focusing on facilitating the development and dissemination of economic evaluations and HTAs across jurisdictions include: the CEA Registry by the Centre for the Evaluation of Value and Risk in health, GEAR, and Decide-Health Decision Hub. The publication of open access health economic models by HTA agencies could also help identify CEAs of interest or initiate more readily available model to be adapted and populated with local inputs. Another initiative is the Regional Database of Health Technology Assessment Reports in Americas (RedETSA) that publishes joint HTA reports for Latin America and the Caribbean [63]. From an evidence modelling perspective, such regional collaborations could lead to developing models which allow for a degree of similarity between countries when estimating both clinical and economic net benefit, as the health care systems are more likely to be comparable. The HTA guidance published by INEAS could be used as a steppingstone in the region, providing a useful reference case to neighbouring countries-e.g. Morocco, Algeriawishing to implement similar HTA frameworks.

Moreover, a broader discussion on how to best incorporate issues of affordability and acceptability to health care providers and patients, especially for HTA agencies aiming to assess costly health interventions already in routine care, is warranted [ $\left.{ }^{64}\right]$. For example, the delivery of healthcare and the management of key diseases can be very different between LMICs and HICs, raising issues about patient co-payments and catastrophic out of pocket expenditures for households. Trastuzumab is an interesting case study to explore the use of HTA in LMICs; as the affordability of trastuzumab is highly topical and has recently been investigated in Iran, Latin America, and Sub Sahara Africa $\left.{ }^{31,65,66}\right]$. Work from Barrett et al. in the UK suggests the opportunity cost of trastuzumab could be considerable, as compared to other treatments for breast cancer; how this extends to other cancer medicines, or to other disease areas would merit further consideration [64,67]. However, the availability of trastuzumab biosimilars at 
substantially lower costs is likely to not only impact the cost-effectiveness of trastuzumab but its use and marketing in Tunisia and other LMICs.

Lastly, better reporting is an efficient way of improving the methodological quality of an HTA without necessarily adding to the resource burden of such assessments. Similarly, pre-defining a HTA protocol, data requirements, and preferred methods for HTA in a tailored methods guide produced by local HTA agency would provide further clarity and consistency to future assessments. In our case study, we highlight a number of instances were HTA elements were not substantiated enough or not reported by INEAS. However, it is likely that these were captured or discussed and could have been included, even in Appendix, for completeness and in line with best HTA practice, such as search strategies and data extraction, psychological, social, and ethical considerations, or organizational and professional implications.

A stated caveat of our review is that specific issues surrounding the appraisal of new health technologies in LMICs, intrinsically linked to HTA, were not considered. Learnings from other countries demonstrate that understanding how evidence generated during the HTA process is translated into resource allocation decisions, and how HTA is implemented as part of a wider health policy change, particularly in LMICs, is critical $\left.{ }^{57}\right]$. Decisionmaking regarding resource allocation to specific healthcare technologies leads to inevitable 'trade-offs' and these may be contentious, if restricting funding and access to potentially useful, cost-effective treatments [ $\left.{ }^{68}\right]$. It is important that the process used to arrive at such decisions is seen as being socially just, otherwise both individual decisions and the evaluation process itself are likely to be contested. In this respect, although stakeholder engagement may be perceived as 'slowing down' and increasing the 'cost' of HTA, it is an important component of a socially just decision-making process and may contribute to the acceptance and increase the impact of HTA based decision-making [ $\left.{ }^{69}\right]$. The 2020 roadmap for systematic priority setting and HTA, developed by Management Sciences for Health (MSH) and the USAID Medicines, Technologies, and Pharmaceutical Services (MTaPS) Program, echoes a number of our recommendations for the implementation of HTA in LMICs. In addition to identifying key challenges for LMICs, this roadmap also provides tools and approaches to support HTA efforts and help navigate the process of institutionalising HTA-"from agenda setting through formulation, adoption/implementation to impact evaluation" [ $\left.{ }^{68}\right]$.

Our critical review compared the approach and methods used by INEAS in their assessment of trastuzumab to a number of published HTA methods guidelines. However, using traditional HTA elements and methods, without consideration for capacity and resource constraints, as a benchmark for 'best practice' in LMICs is inconsistent. It may be more appropriate to focus on how useful an assessment is, given an agency's resource constraints. Luz et al. find that the lack of standard practice that is relevant to LMICs was frequently reported as an 
issue by countries surveyed regarding the conduct, reporting and use of economic evaluations $\left[{ }^{10}\right]$. Despite challenges, it is promising that the core principles underpinning good HTA practice are endorsed by newly established HTA agencies such as INEAS [4,9,24]; and that ongoing research and global initiatives are continuously extending and adapting HTA guidelines to advance HTA practice in both HICs and LMICs [2,68,70]. 


\section{References}

1. World Health Organization (WHO) 67. Health Intervention and Technology Assessment in Support of Universal Health Coverage. EB134/30.; 2014. https://apps.who.int/iris/handle/10665/162870

2. O'Brien N, Li R, Isaranuwatchai W, et al. How can we make better health decisions: a Best Buy for all? Gates Open Res. 2020;3:1543. doi:10.12688/gatesopenres.13063.2

3. Chalkidou K, Marten R, Cutler D, et al. Health technology assessment in universal health coverage. The Lancet. 2013;382(9910):e48-e49. doi:10.1016/S0140-6736(13)62559-3

4. Tantivess S, Chalkidou K, Tritasavit N, Teerawattananon Y. Health Technology Assessment capacity development in low- and middle-income countries: Experiences from the international units of HITAP and NICE. F1000Res. 2017;6:2119. doi:10.12688/f1000research.13180.1

5. United Nations. Adopting Consensus Text, General Assembly Encourages Member States to Plan, Pursue Transition of National Health Care Systems towards Universal Coverage | Meetings Coverage and Press Releases. Published December 12, 2012. Accessed April 29, 2020. https://www.un.org/press/en/2012/ga11326.doc.htm

6. Oortwijn W, Mathijssen J, Banta D. The role of health technology assessment on pharmaceutical reimbursement in selected middle-income countries. Health Policy. 2010;95(2-3):174-184. doi:10.1016/j.healthpol.2009.12.008

7. Announcing the new definition of HTA! INAHTA. Published May 13, 2020. Accessed October 4, 2020. https://www.inahta.org/2020/05/announcing-the-new-definition-of-hta/

8. Torbica A, Fornaro G, Tarricone R, Drummond MF. Do Social Values and Institutional Context Shape the Use of Economic Evaluation in Reimbursement Decisions? An Empirical Analysis. Value in Health. 2020;23(1):1724. doi:10.1016/j.jval.2019.11.001

9. Adeagbo CU, Rattanavipapong W, Guinness L, Teerawattananon Y. The Development of the Guide to Economic Analysis and Research (GEAR) Online Resource for Low- and Middle-Income Countries' Health Economics Practitioners: A Commentary. Value in Health. 2018;21(5):569-572. doi:10.1016/j.jval.2017.10.003

10. Luz A, Santatiwongchai B, Pattanaphesaj J, Teerawattananon Y. Identifying priority technical and contextspecific issues in improving the conduct, reporting and use of health economic evaluation in low- and middleincome countries. Health Res Policy Sys. 2018;16(1):4. doi:10.1186/s12961-018-0280-6

11. Bannour I, Briki R, Zrairi F, et al. Breast cancer in the Maghreb : epidemiology and control strategies. Review. Tunis Med. 2018;96(10-11):658-664.

12. Missaoui N, Jaidene L, Abdelkrim SB, et al. Breast cancer in Tunisia: clinical and pathological findings. Asian Pac J Cancer Prev. 2011;12(1):169-172.

13. Bayoudh L, Afrit M, Daldoul O, Zarrad M, Boussen H. [Trastuzumab (herceptin) for the medical treatment of breast cancer]. Tunis Med. 2012;90(1):6-12.

14. Cherif A, Dhaouadi S, Osman M, Hsairi M. Breast Cancer burden in Tunisia: situation in 2017 and projections by 2030. European Journal of Public Health. 2019;29(Supplement_4):ckz186.232. doi:10.1093/eurpub/ckz186.232

15. Jameleddine M, Grati H, Jebali MC. Le trastuzumab dans le traitement du cancer du sein HER2 positif au stade précoce et localement avancé. Avis d'Evaluation des Technologies de Santé. Published online July 2018. http://www.ineas.tn/sites/default/files/trastuzumab_premier_rapport_ets_ineas_-ilovepdf-compressed.pdf

16. World Health Organization (WHO) RO for the EM. Tunisia: Medicine prices, availability, affordability and price components. 2010;(WHO-EM/EDB/100/E). https://apps.who.int/iris/handle/10665/116638

17. World Health Organization (WHO). Tunisia: Health Systems Profile. Key health system indicators. Published 2013. Accessed October 11, 2020. https://applications.emro.who.int/docs/Country_profile_2013_EN_15402.pdf 
18. Busse R, Orvain J, Velasco M, Perleth M, Drummond M. Best practice in undertaking and reporting health technology assessments. Published online 2002:62.

19. Drummond MF, Jefferson TO. Guidelines for authors and peer reviewers of economic submissions to the BMJ. BMJ. 1996;313(7052):275-283. doi:10.1136/bmj.313.7052.275

20. National Institute for Health and Care Excellence (NICE). Guide to the Methods of Technology Appraisal.; 2013. Accessed April 9, 2019. https://www.nice.org.uk/process/pmg9/chapter/foreword

21. Pichon-Riviere A, Soto N, Augustovski F, Rey-Ares L, Garcia-Marti S, Sampietro-Colom L. Good Practices in Application of Health Technology Assessment For Decision-Making Worldwide. Health Technology Assessment international (HTAi). Published online 2016:25.

22. Centre for Reviews and Dissemination, ed. CRD's Guidance for Undertaking Reviews in Healthcare. 3. ed. York Publ. Services; 2009.

23. Edejer TT-T, World Health Organization, eds. Making Choices in Health: WHO Guide to Cost-Effectiveness Analysis. World Health Organization; 2003.

24. Claxton K, Revill P, Sculpher M, Wilkinson T, Cairns J, Briggs A. The Gates Reference Case for Economic Evaluation. Published online April 2014. http://www.idsihealth.org/wp-content/uploads/2016/05/GatesReference-case-what-it-is-how-to-use-it.pdf

25. Indonesian Health Technology, Assessment Committee (InaHTAC). Health Technology Assessment (HTA) Guideline. Published online 2017. http://www.gear4health.com/uploads/files/file-15-5a441f38c8992.pdf

26. Chaikledkaew U, Kittrongsiri K. Guidelines for health technology assessment in Thailand (second edition)--the development process. J Med Assoc Thai. 2014;97 Suppl 5:S4-9.

27. la Société Tunisienne d'Oncologie Médicale. Référentiels et Recommandations de la Société Tunisienne d'Oncologie Médicale. Published online 2016.

28. Scottish Intercollegiate Guidelines Network, Scotland, Healthcare Improvement Scotland. Treatment of Primary Breast Cancer: A National Clinical Guideline.; 2013. https://www.sign.ac.uk/assets/sign134.pdf

29. National Institute for Health and Care Excellence (NICE). Early and locally advanced breast cancer: diagnosis and management. Published online July 18, 2018. https://www.nice.org.uk/guidance/ng101

30. Moja L, Tagliabue L, Balduzzi S, et al. Trastuzumab containing regimens for early breast cancer. Cochrane Breast Cancer Group, ed. Cochrane Database of Systematic Reviews. Published online April 18, 2012. doi:10.1002/14651858.CD006243.pub2

31. Pichon-Riviere A, Garay OU, Augustovski F, et al. Implications of global pricing policies on access to innovative drugs: the case of trastuzumab in seven Latin American countries. Int J Technol Assess Health Care. 2015;31(1-2):2-11. doi:10.1017/S0266462315000094

32. Piccart-Gebhart MJ, Procter M, Leyland-Jones B, et al. Trastuzumab after Adjuvant Chemotherapy in HER2Positive Breast Cancer. N Engl J Med. 2005;353(16):1659-1672. doi:10.1056/NEJMoa052306

33. Calvert MJ, Freemantle N, Cleland JGF. The impact of chronic heart failure on health-related quality of life data acquired in the baseline phase of the CARE-HF study. European Journal of Heart Failure. 2005;7(2):243-251. doi:10.1016/j.ejheart.2005.01.012

34. Lidgren M, Wilking N, Jönsson B, Rehnberg C. Health related quality of life in different states of breast cancer. Qual Life Res. 2007;16(6):1073-1081. doi:10.1007/s11136-007-9202-8

35. Drummond MF, Schwartz JS, Jönsson B, et al. Key principles for the improved conduct of health technology assessments for resource allocation decisions. Int J Technol Assess Health Care. 2008;24(03):244-258. doi:10.1017/S0266462308080343 
36. World Health Organization (WHO) 67. WHO | Pilot Procedure for Prequalification of Biotherapeutic Products and Similar Biotherapeutic Products. WHO. Published 2018. Accessed October 12, 2020.

http://www.who.int/medicines/regulation/biotherapeutic_products/en/

37. Chaiyakunapruk N, Saokaew S, Sruamsiri R, Dilokthornsakul P. Systematic review and network meta-analysis in health technology assessment. J Med Assoc Thai. 2014;97 Suppl 5:S33-42.

38. Burstein HJ, Griggs JJ, Prestrud AA, Temin S. American Society of Clinical Oncology Clinical Practice Guideline Update on Adjuvant Endocrine Therapy for Women With Hormone Receptor-Positive Breast Cancer. Journal of Oncology Practice. 2010;6(5):6.

39. Cardoso F, Costa A, Norton L, et al. ESO-ESMO 2nd international consensus guidelines for advanced breast cancer (ABC2). The Breast. 2014;23(5):489-502. doi:10.1016/j.breast.2014.08.009

40. Cardoso F, Costa A, Senkus E, et al. 3rd ESO-ESMO International Consensus Guidelines for Advanced Breast Cancer (ABC 3). Annals of Oncology. 2017;28(1):16-33. doi:10.1093/annonc/mdw544

41. Cardoso F, Kyriakides S, Ohno S, et al. Early breast cancer: ESMO Clinical Practice Guidelines for diagnosis, treatment and follow-up. Annals of Oncology. 2019;30(8):1194-1220. doi:10.1093/annonc/mdz173

42. Philips Z, Bojke L, Sculpher M, Claxton K, Golder S. Good Practice Guidelines for Decision-Analytic Modelling in Health Technology Assessment: A Review and Consolidation of Quality Assessment. PharmacoEconomics. 2006;24(4):355-371. doi:10.2165/00019053-200624040-00006

43. Hay JW, Smeeding J, Carroll NV, et al. Good research practices for measuring drug costs in cost effectiveness analyses: issues and recommendations: the ISPOR Drug Cost Task Force report--Part I. Value Health. 2010;13(1):3-7. doi:10.1111/j.1524-4733.2009.00663.x

44. Drummond $\mathrm{M}$, Augustovski $\mathrm{F}$, Kaló $Z$, et al. Challenges faced in transferring economic evaluations to middle income countries. Int J Technol Assess Health Care. 2015;31(6):442-448. doi:10.1017/S0266462315000604

45. Husereau D, Drummond M, Petrou S, et al. Consolidated Health Economic Evaluation Reporting Standards (CHEERS)--explanation and elaboration: a report of the ISPOR Health Economic Evaluation Publication Guidelines Good Reporting Practices Task Force. Value Health. 2013;16(2):231-250. doi:10.1016/j.jval.2013.02.002

46. Riewpaiboon A. Measurement of Costs for Health Economic Evaluation. JOURNAL OF THE MEDICAL ASSOCIATION OF THAILAND. 2014;97(5):17.

47. Woods B, Revill P, Sculpher M, Claxton K. Country-Level Cost-Effectiveness Thresholds: Initial Estimates and the Need for Further Research. Value Health. 2016;19(8):929-935. doi:10.1016/j.jval.2016.02.017

48. Ochalek J, Lomas J, Claxton K. Estimating health opportunity costs in low-income and middle-income countries: a novel approach and evidence from cross-country data. BMJ Glob Health. 2018;3(6):e000964. doi:10.1136/bmjgh-2018-000964

49. Bertram MY, Lauer JA, De Joncheere K, et al. Cost-effectiveness thresholds: pros and cons. Bull World Health Organ. 2016;94(12):925-930. doi:10.2471/BLT.15.164418

50. Goeree R, He J, O'Reilly D, et al. Transferability of health technology assessments and economic evaluations: a systematic review of approaches for assessment and application. Clinicoecon Outcomes Res. 2011;3:89-104. doi:10.2147/CEOR.S14404

51. Fan H, Song F. An assessment of randomized controlled trials (RCTs) for non-communicable diseases (NCDs): more and higher quality research is required in less developed countries. Sci Rep. 2015;5(1):13221. doi:10.1038/srep13221

52. Riewpaiboon A. Measurement of costs for health economic evaluation. J Med Assoc Thai. 2014;97 Suppl 5:S17-26. 
53. Acurcio FA, Perini E, Magalhães SMS, et al. Analysis of medical prescriptions dispensed at health centers in Belo Horizonte, Minas Gerais, Brazil. Cad Saúde Pública. 2004;20(1):72-79. doi:10.1590/S0102$311 \times 2004000100021$

54. Acurcio F de A, Cesar CC, Guimarães MDC. Health care utilization and survival among patients with AIDS in Belo Horizonte, Minas Gerais, Brazil. Cad Saúde Pública. 1998;14(4):811-820. doi:10.1590/S0102$311 \mathrm{X} 1998000400023$

55. Boccolini CS, de Souza Junior PRB. Inequities in Healthcare utilization: results of the Brazilian National Health Survey, 2013. Int J Equity Health. 2016;15(1):150. doi:10.1186/s12939-016-0444-3

56. Abera Abaerei A, Ncayiyana J, Levin J. Health-care utilization and associated factors in Gauteng province, South Africa. Global Health Action. 2017;10(1):1305765. doi:10.1080/16549716.2017.1305765

57. Yuba TY, Novaes HMD, de Soárez PC. Challenges to decision-making processes in the national HTA agency in Brazil: operational procedures, evidence use and recommendations. Health Res Policy Sys. 2018;16(1):40. doi:10.1186/s12961-018-0319-8

58. Jakupi A, Godman B, Martin A, Haycox A, Baholli I. Utilization and Expenditure of Anti-cancer Medicines in Kosovo: Findings and Implications. PharmacoEconomics Open. 2018;2(4):423-432. doi:10.1007/s41669-0170066-8

59. Kivoto PM, Mulaku M, Ouma C, et al. Clinical and Financial Implications of Medicine Consumption Patterns at a Leading Referral Hospital in Kenya to Guide Future Planning of Care. Front Pharmacol. 2018;9:1348.

doi:10.3389/fphar.2018.01348

60. Jeffery M, Chi Y-L, Stewart M. iDSI Health Technology Assessment Toolkit. Published online September 25, 2018. Accessed April 30, 2020. https://f1000research.com/documents/7-1545

61. MacQuilkan K, Baker P, Downey L, et al. Strengthening health technology assessment systems in the global south: a comparative analysis of the HTA journeys of China, India and South Africa. Global Health Action. 2018;11(1):1527556. doi:10.1080/16549716.2018.1527556

62. Hollingworth S, Gyansa-Lutterodt M, Dsane-Selby L, et al. Implementing health technology assessment in Ghana to support universal health coverage: building relationships that focus on people, policy, and process. Int J Technol Assess Health Care. Published online November 28, 2019:1-4. doi:10.1017/S0266462319000795

63. Pichon-Riviere A, Glujovsky D, Garay OU, Augustovski F, Ciapponi A. Economic evaluation of oxytocin in Uniject ${ }^{\mathrm{TM}}$ injection system versus standard use of oxytocin for the prevention of postpartum hemorrhage in the active management of the third stage of labor in Latin America and the Caribbean. Technical report IECS N ${ }^{\circ}$ 12. Published online December 2013. https://www.paho.org/en/documents/economic-evaluation-oxytocinunijecttm-injection-system-versus-standard-use-oxytocin

64. World Health Organization (WHO) 67. Pricing of cancer medicines and its impacts. ACCESS TO MEDICINES, VACCINES AND PHARMACEUTICALS. TECHNICAL REPORT. Published 2018. Accessed October 12, 2020. https://apps.who.int/iris/bitstream/handle/10665/277190/9789241515115-eng.pdf?ua=1

65. Ansaripour A, Uyl-de Groot C, Redekop W. What is an Efficient and Affordable Trastuzumab Therapy in a Middle-Income Country? - Adjuvant Therapy with Trastuzumab in Management of Early HER2-Positive Breast Cancer in Iran. Value in Health. 2016;19(7):A721. doi:10.1016/j.jval.2016.09.2147

66. Gershon N, Berchenko Y, Hall PS, Goldstein DA. Cost effectiveness and affordability of trastuzumab in subSaharan Africa for early stage HER2-positive breast cancer. Cost Eff Resour Alloc. 2019;17(1):5. doi:10.1186/s12962-019-0174-7

67. Barrett A, Roques T, Small M, Smith RD. How much will Herceptin really cost? BMJ. 2006;333(7578):11181120. doi:10.1136/bmj.39008.624051.BE

68. Management Sciences for Health (MSH). A Roadmap for Systematic Priority Setting and Health Technology Assessment (HTA): A Practical Guide for Policy Action in Low- And Middle-Income Countries. | Management 
Sciences for Health. Published 2020. Accessed October 12, 2020. /resources/a-roadmap-for-systematicpriority-setting-and-health-technology-assessment-hta-a-practical

69. Dolan P. Modeling valuations for EuroQol health states. Med Care. 1997;35(11):1095-1108. doi:10.1097/00005650-199711000-00002

70. Kristensen FB, Husereau D, Huić M, et al. Identifying the Need for Good Practices in Health Technology Assessment: Summary of the ISPOR HTA Council Working Group Report on Good Practices in HTA. Value in Health. 2019;22(1):13-20. doi:10.1016/j.jval.2018.08.010 